feelings settle very quickly and majority of them feel improvement in their key respiratory symptoms. Contrary to common perception majority will be happy to have treatment again. Patients felt that a detailed explanation and counselling before starting NIV improves compliance and successful outcome from NIV.

\section{P224 WHY ARE WE FAILING IN THE UK IN NON-INVASIVE VENTILATION (NIV) AND ACUTE EXACERBATIONS OF COPD (AECOPD)? REVIEW OF OUR LOCAL PRACTISE}

doi:10.1136/thoraxjnl-2012-202678.285

KM Protheroe, R Bentley, TE Sams, S Parker, R Sayers, J Taylor, JW Killen, HJ Curtis. Gateshead Health NHS Foundation Trust, Gateshead, UK

Introduction and Objectives Ward based NIV is proven treatment in AECOPD with type II respiratory failure with $\mathrm{pH}$ 7.25- 7.35.(1) Increasingly this modality is being used out with the trial evidence.

The RCP audit of real world practise showed concerning results: failure or delay to deliver NIV and increaced mortality in NIVtreated patients compared to equally severe patients managed without NIV (26\% vs $14 \%)$.(2)

In light of these factors we reviewed our NIV use across our hospital. Did our local service need improvement?

Methods We audited 4 months of emergency department admissiond in late 2011 to 2012, ward based NIV care in February-March 2011 and February-March 2012 using the BTS audit tool and critical care admissions for AECOPD from January 2010 to Decemeber 2011. Results

\section{Emergency Department}

NIV was only considered in $78 \%$ of possible patients and only given in $37 \%$. Significant delays were seen in starting NIV; median 357 minutes (range 138-1366).

\section{Ward-based NIV}

In 2011 overall mortality was 33\%, however patients with pH 7.257.35 mortality was $11 \%$, matching the landmark trial outcomes.(1) If $\mathrm{pH}$ was $<7.25$ mortality was $80 \%$. In 2012 oxygen toxicity contributed to acidosis is $33 \%$ of patients and overall mortality was $40 \%$

\section{Critical Care Department (CCD)}

Time to respiratory support was a median of 4 hours. $31 \%$ of patients required invasive ventilation, this was higher if consolidation was present on CXR ( $p=0.005)$. Overall mortality was $20 \%$, significantly higher if $\mathrm{pH}<7.25$ at any time at $35 \%(\mathrm{p}=0.02)$ and if CXR consolidation was present $(25 \%$ vs 12.5$)$

Conclusions Unfortunately NIV is not commenced in all appropriate patients, delays are common place and NIV is being used in severely ill and very acidotic patients with high mortality outcomes.

Driven by national audit data, this detailed analysis of our practise has allowed us to drive local changes to improve our service including: 24/7 NIV nurse; early involvement with CCD in appropraite patients with $\mathrm{pH}<7.25$ and re-education of staff across the Trust.

\section{References}

1. Plant PK et al. Early use of NIV for AECOPD on general respiratory wards. Lancet 2000; 355:1931-35.

2. Roberts CM et al. Acidosis, NIV and mortality in hospitalised COPD exacerbations. Thorax 2011; 66:43-48.

\section{P225 ROUTES OF DOMICILIARY NON-INVASIVE VENTILATION (NIV) SET-UP}

doi:10.1136/thoraxjnl-2012-202678.286

'S Agarwal, 'M Vaughan, 'C Wharton, 'S Ejiofor, 'B Chakraborty, 'E Gallagher, 'R Mukherjee. 'Birmingham Heartlands Hospital, Birmingham, UK; ${ }^{2}$ School of Mathematics, Univ. of Birmingham, Birmingham, UK
Introduction Domiciliary NIV is being increasingly used to treat chronic ventilatory failure, particularly due to obesity and neuromuscular conditions. In the course of evolution of an NIV unit within an acute hospital, most domiciliary NIVs are set up at the end of an acute episode of admission with hypercapnic acidotic respiratory failure to start with, but overtime, as more at-risk patients come under surveillance for respiratory failure, we hypothesized that a unit supervising domiciliary NIV/Home Mechanical Ventilation is expected to do more elective set-ups.

Methods Comparison of the volume of new domiciliary NIV setups and the elective NIV set-up rate over two 12-month periods: Apr 2005-Mar 2006 (period 1) and Apr 2011-Mar 2012 (period 2) in a dedicated 11-bedded ward-based NIV unit (established: Aug 2004) in a 1000-bedded central England teaching hospital trust, providing domiciliary NIV support to over 200 patients with over 350 under surveillance for respiratory failure.

Results The volume more than doubled from 19 new domiciliary NIV set-ups in period 1 to 39 new domiciliary NIV set-ups in period 2; the elective domiciliary NIV set-up rate increased from $7 / 19$ (36.8\%) to 19/39 (48.7\%) between periods 1 and 2 .

Discussion Over time, both the volume and the elective set-uprate for new domiciliary NIV have gone up. This probably indicates that a larger proportion of people at risk of respiratory failure treatable with NIV are coming under the unit's surveillance and has clearly been associated with the expansion and maturation of the NIV service in our experience. The 'elective domiciliary NIV set-up rate' can therefore be tested as a metric for comparison of centres supervising domiciliary NIV/Home Mechanical Ventilation in this rapidly evolving field.

\section{P226 THE CHANGING FACE OF HOME NIV (NON INVASIVE VENTILATION)}

doi:10.1136/thoraxjnl-2012-202678.287

SV Fletcher, S Ewles, JE Wilkinson. University Hospital Southampton NHS Foundation Trust, Southampton, UK

Introduction The number of patients requiring home NIV for chronic hypercapnic respiratory failure is rising and the indications are changing. This has significant service planning and cost implications.

Methods A retrospective review of the database of all patients established on home NIV since 2004 was conducted. All clinical records from these patients were reviewed. The indication for NIV was classified as thoracic cage abnormalities, neuromuscular disease, OHS (obesity hypoventilation syndrome +/- obstructive sleep apnoea), COPD (chronic obstructive pulmonary disease), $\mathrm{CF}$ (cystic fibrosis) and ILD (interstitial lung disease)/other. The date of death was gained from the internal hospital records (eDocs) and through the NHS portal with the use of individual NHS numbers.

Results There were 286 patients established on home NIV between 2004 and 2012, 162 were male, the overall mortality was 29\%.

There has been over a seven fold increase in the yearly prevalence of patients requiring home NIV and the indication for its use is changing over time (figure 1). The proportion of patients with thoracic cage abnormalities is reducing from $25 \%$ in 2004 to $11 \%$ in 2012. The use of NIV for obesity hypoventilation syndrome has increased 10 fold since 2004. This hospital is the regional neurosciences referral unit which may explain the large number of patients requiring NIV for neuromuscular disease, $45 \%$ of which had motor neurone disease.

In 2004, the cost of setup with an NIV machine and consumable for a all new patients, plus the cost of consumables for those established on NIV was estimated to be $£ 73,000$; whereas this cost in 2011 was estimated to be $£ 308,500$ (incl VAT). This does not include the cost of personnel. 